\title{
Preparation and Properties of Carboxymethyl Chitosan/Alginate/Tranexamic Acid Composite Films
}

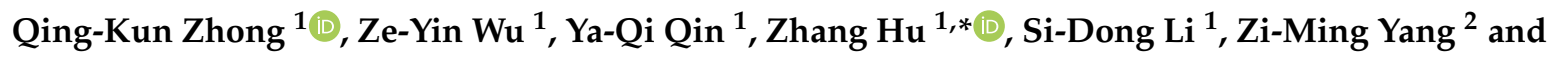 \\ Pu-Wang $\mathrm{Li}^{2, *}$ \\ 1 School of Chemistry and Environmental Science, Guangdong Ocean University, Zhanjiang 524088, China; \\ 13924405077@163.com (Q.-K.Z.); guazi.wu@foxmail.com (Z.-Y.W.); Qmumuyi@163.com (Y.-Q.Q.); \\ sidongligdou@163.com (S.-D.L.) \\ 2 Agricultural Product Processing Research Institute, Chinese Academy of Tropical agricultural Sciences, \\ Zhanjiang 524001, China; yangziming2004@163.com \\ * Correspondence: huzhangqyx@126.com (Z.H.); puwangli@163.com (P.-W.L.); Tel.: +86-759-238-3300 (Z.H.)
}

Received: 4 December 2018; Accepted: 4 January 2019; Published: 8 January 2019

Abstract: In this study, the porous composite films of carboxymethyl chitosan/alginate/tranexamic acid were fabricated, with calcium chloride as the crosslinking agent and glycerin as a plasticizer. The composite films were characterized by scanning electron microscopy (SEM) and Fourier transform infrared (FTIR) spectroscopy. The properties of the composite films, including water absorption, air permeability, and cumulative release rate, were tested. In addition, their hemostatic performance was evaluated. The results showed that the appearance of the films with good adhesion was smooth and porous. FTIR showed that chemical crosslinking between carboxymethyl chitosan and sodium alginate was successful. The excellent cumulative release of tranexamic acid in the composite films $(60-80 \%)$ gives the films a significant procoagulant effect. This has good prospects for the development of medical hemostasis materials.

Keywords: carboxymethyl chitosan; sodium alginate; tranexamic acid; composite films; hemostatic activity

\section{Introduction}

The skin is the first barrier of the human body for defense against external stimuli and damage. Damage caused by mechanical factors can lead to microbial invasion, to loss of protein, water, and blood volume, and even to death [1,2]. Therefore, the development of excellent films for skin trauma and rapid hemostasis is key to treatment, which is closely related to human health [3].

Chitosan (CS) is a product obtained by the deacetylation of chitin, which may be extracted from shrimp and crab shells [4]. Owing to its excellent biocompatibility, biodegradability, non-toxicity, and various physiological functions such as hemostasis, bacteriostatic, anti-cancer, and lipid-lowering [5,6], chitosan has been extensively used in biomedicine. Carboxymethyl chitosan (CMCS) is a kind of secondary derivative obtained by the carboxymethylation of chitosan [7]. Due to the coexistence of $-\mathrm{NH}_{2}$ and $-\mathrm{COOH}, \mathrm{CMCS}$ has better biocompatibility, moisture absorption, antibacterial, and film-forming properties compared to chitosan $[8,9]$. Sodium alginate (SA) is a natural polysaccharide extracted from kelp or seaweed. As a natural wound repairing material, sodium alginate has good thickening, flocculation, and chelating properties [10]. Due to its unique film-forming properties and non-toxic degradability, it is widely used as a gel film and clinical medical dressing which has healing effects on burns, piercing wounds, and deep ulcer bleeding [11,12]. Tranexamic acid (TA) is a synthetic antifibrinolytic drug which has been used clinically in the treatment of bleeding caused by fibrinolysis [13-15]. Its structure is similar to that of lysine, which inhibits the cleavage of fibrin 
clots by competitively inhibiting the binding of fibrin lysine to plasmin, thereby producing hemostasis. However, TA has a short half-life and low bioavailability. It is an effective approach to controlled drug release by using polymer as a carrier. To the best of our knowledge, the combination of tranexamic acid and polymer matrix as a new hemostatic material has been seldom studied.

In this paper, sodium alginate and carboxymethyl chitosan were used as the carrier matrix and a hemostatic active ingredient tranexamic acid was added to prepare the composite films which had good sustained release effects. The composite films are expected to be used for clinical hemostasis.

\section{Materials and Methods}

\subsection{Materials}

Carboxymethyl chitosan, sodium alginate, tranexamic acid, glycerin, and anhydrous calcium chloride were all purchased from Sinopharm Chemical Reagent Co., Ltd. (Shanghai, China). Unless otherwise specified, all reagents were of analytical grade.

\subsection{Fabrication of the Composite Films}

Solutions with a total volume of $6 \mathrm{~mL}$ of SA $(2 \%, w / v)$ and CMCS $(2 \%, w / v)$ were mixed at volume ratios of 1:1, 1:2, 2:1, 1:5, 5:1, pure CMCS, and pure SA, and were recorded as samples 1\#, 2\#, $3 \#, 4 \#, 5 \#, 6 \#$, and $7 \#$, respectively. After being stirred fully, $0.5 \% \mathrm{CaCl}_{2}$ solution $(2 \mathrm{~mL})$ was added to crosslink and the mixture was frozen overnight. The mixture was washed repeatedly with absolute ethanol three times and the matrix films were formed. The matrix films were then put into a solution of tranexamic acid $(0.01 \mathrm{~g} / \mathrm{mL})-10 \%$ glycerol and soaked for $30 \mathrm{~min}$. The composite films were obtained by lyophilization at $-60^{\circ} \mathrm{C}$ for $24 \mathrm{~h}$.

\subsection{Appearance Observation}

The appearance of the composite films was recorded with a digital camera and a scanning electron microscope (SEM) (Hitachi S-4800, Tokyo, Japan). The pretreatment of samples for observation under the SEM was the process of gold spray. The conditions of determination were a working voltage of $1 \mathrm{kV}$ and a distance of $10.3 \mathrm{~mm}$.

\subsection{Measurement of Fourier Transform Infrared (FTIR)}

A Spectrum 100 Fourier transform infrared spectrometer (PerkinElmer, Waltham, MA, USA) was used to measure the samples. The films were directly used for an attenuated total reflection (ATR) accessory. The infrared spectrum was recorded over wave numbers ranging from $4000 \mathrm{~cm}^{-1}$ to $450 \mathrm{~cm}^{-1}$.

\subsection{Determination of Water Absorption Rate}

The composite films were cut into rectangular pieces and weighed. The rectangular films were placed into a beaker containing distilled water for $3 \mathrm{~h}$ at room temperature and then taken out. The water on the surface of the films was removed with filter paper and the films were weighed again. Tests were performed in triplicate and the results were expressed as mean \pm standard deviation. The formula for the water absorption rate was

$$
\text { Water absorption rate }(\%)=\frac{m_{1}-m_{0}}{m_{1}} \times 100 \%
$$

where, $m_{0}$ and $m_{1}$ were the mass of films before and after soaking, respectively. 


\subsection{Determination of Air Permeability}

Two same-size Erlenmeyer flasks, each containing $100 \mathrm{~mL}$ distilled water, were weighed $\left(m_{1}\right)$. One was sealed with the composite films and the other left untreated and set as the blank control. They were placed overnight in an environment with a humidity of $43 \%$ (saturated $\mathrm{Na}_{2} \mathrm{CO}_{3}$ solution) at room temperature. The films were then removed and the flask which had contained them was weighed again $\left(m_{2}\right)$. The blank control flask was weighed as $m_{3}$. A fresh-keeping film sold commercially was used as a positive control. The formula for the air permeability was

$$
\text { Air permeability }(\%)=\left(m_{1}-m_{2}\right) /\left(m_{1}-m_{3}\right) \times 100 \%
$$

where $m_{1}-m_{2}$ and $m_{1}-m_{3}$ were the losses of water in the experimental group and in the control group, respectively.

\subsection{Determination of Cumulative Release Percent}

The composite films (50 mg) were placed in an Erlenmeyer flask and the volume was set to $50 \mathrm{~mL}$ using distilled water. An amount of the solution $(1 \mathrm{~mL})$ was removed from the flask and the same volume of distilled water was replenished at designated times $(5 \mathrm{~min}, 10 \mathrm{~min}, 30 \mathrm{~min}, 1 \mathrm{~h}, 3 \mathrm{~h}$ and $24 \mathrm{~h}$ ). The absorbance of the solution was measured using spectrophotometry (UV-1800, Jinghua Technology, Shanghai, China) at $220 \mathrm{~nm}$ and the content of tranexamic acid was calculated according to the measured standard curve formula $\left(C=54.35 \mathrm{~A}+1.76, R^{2}=0.99503\right)$. The cumulative release percent $(C R P \%)$ was calculated as

$$
C R P \%=\frac{C_{j} \times V_{\text {total }}+\sum_{i=0}^{j-1} C_{i} V_{\text {taken }}}{m} \times 100 \%
$$

where, $C_{i}$ and $C_{j}$ were the concentrations of tranexamic acid at the moments $i$ and $j$, respectively, in $\mu \mathrm{g} / \mathrm{mL} ; V_{\text {taken }}$ and $V_{\text {total }}$ were the volumes of the taken and total solutions, respectively, in $\mathrm{mL}$; and $m$ was the total mass of tranexamic acid in the composite films.

\subsection{In Vitro Clotting Time}

The composite films $(0.20 \mathrm{~g})$ were placed in a test tube and kept at $37^{\circ} \mathrm{C}$. Fresh anticoagulated rabbit blood ( $1 \mathrm{~mL} /$ tube) was added and timing subsequently started. The tube was tilted every $15 \mathrm{~s}$ and timing continued until the blood clotted. A blank control and a positive control (Yunnan Baiyao, $1 \mathrm{~g} / \mathrm{mL}$ ) were set. The study was performed in accordance to the National Research Council Guide for the Care and Use of Laboratory Animals, and the protocol was approved by the Ethics Committee of Guangdong Medical University (Certificate No. SYXK20150147).

\section{Results and Discussion}

\subsection{Morphological Observation}

The composite films of carboxymethyl chitosan/alginate/tranexamic acid were fabricated successfully. The film 1\# was pale yellow and had a porous structure which was favorable for adsorbing liquid (Figure 1a). It can be seen from Figure 1b that tranexamic acid was loaded into the composite films through crystallization to increase the hemostatic property of the composite films. The appearances of other composite films (2-5\#) were similar. 


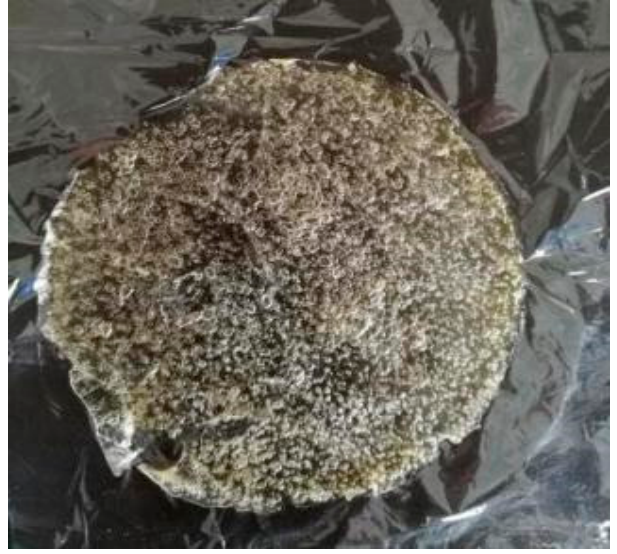

(a)

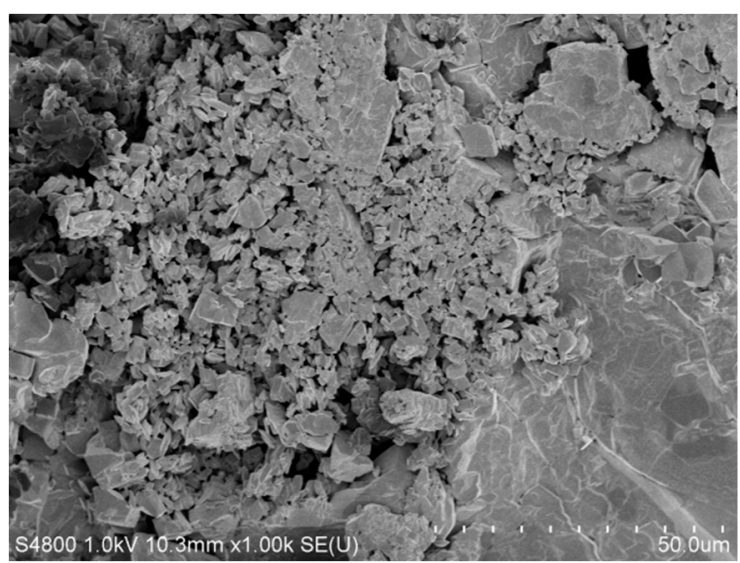

(b)

Figure 1. Appearances of the composite porous film 1\# of carboxymethyl chitosan/alginate/tranexamic acid: (a) optical view, showing a porous structure and (b) scanning electron microscopy (SEM) micrographs, showing the loaded tranexamic acid.

\subsection{Water Absorption}

High water absorbency is one of the important indicators for biological films. The water absorption results of the films are shown in Table 1. It can be seen that the pure CMCS film (6\#) had the worst water absorption, while the pure SA film (7\#) had the best. All the composite films (1-5\#) had good water absorbency, especially sample 4\#. Hydrophilicity is an important characteristic property of hemostatic biomaterials [16]. Though the content of tranexamic acid is the same, the ratio of carboxymethyl chitosan to sodium alginate is different. Water absorption is affected by the cross-linking density which is related to the amount of carboxyl groups [17]. It may be that sodium alginate has more carboxyl groups and better hydrophilicity, while the porous structure of the cross-linked composite films is more compact, which makes the water absorption slightly weaker [11].

Table 1. Water absorption rates of the composite porous films.

\begin{tabular}{cccc}
\hline Film & $\boldsymbol{m}_{\mathbf{0}}(\mathbf{g})$ & $\boldsymbol{m}_{\mathbf{1}}(\mathbf{g})$ & Water Absorption Rate (\%) \\
\hline 1 & 0.013 & 0.165 & $1169 \pm 21$ \\
2 & 0.015 & 0.216 & $1340 \pm 17$ \\
3 & 0.015 & 0.202 & $1246 \pm 19$ \\
4 & 0.015 & 0.290 & $1833 \pm 24$ \\
5 & 0.015 & 0.170 & $1033 \pm 15$ \\
6 & 0.019 & 0.172 & $805 \pm 16$ \\
7 & 0.016 & 0.325 & $1931 \pm 20$ \\
\hline
\end{tabular}

\subsection{Air Permeability}

Air permeability plays an important role in medical wound healing films, and high air permeability is beneficial for wound healing. Cross-linking density has an effect on air permeability. As the cross-linking degree increases, the pore structure becomes dense and air convection is reduced. As a result, the permeability decreases [18]. Without doubt, changes in air permeability are mainly affected by the porosity of the material. The higher the porosity is, the better the gas permeability [19]. The results of air permeability are shown in Figure 2. The fresh-keeping film (Lead Mens ${ }^{\circledR}$, Zhongshan Limeng Aluminum-Plastic Composite Packaging Co., Ltd., Zhongshan, China) sold commercially (the control) had poor permeability, while all the composite films (1-5\#) had excellent air permeability ranging from $80 \%$ to $86 \%$, indicating that the prepared composite films met the high permeability requirements of medical wound dressings. 


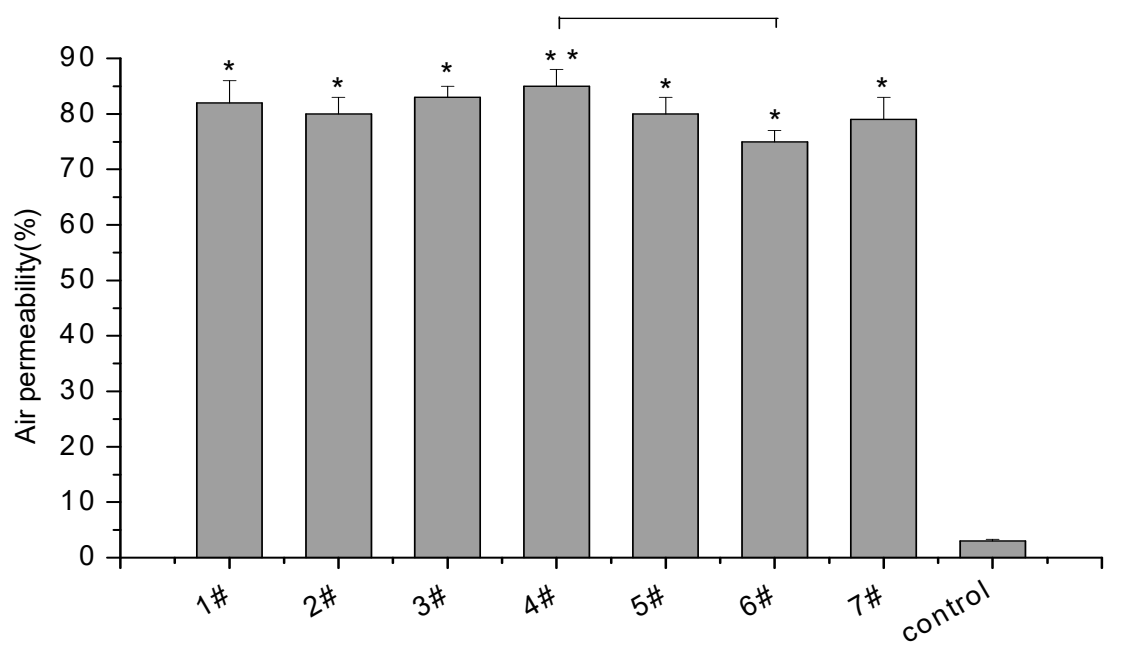

Figure 2. Air permeability of the composite films.

\subsection{FTIR Analysis}

With regard to the FTIR spectrum of CMCS (Figure 3a), it can be concluded that the peak at $3417 \mathrm{~cm}^{-1}$ corresponded to stretching vibrations of the hydroxyl and amino groups, and that the peak at $2963-2847 \mathrm{~cm}^{-1}$ belonged to a $-\mathrm{CH}$ stretching vibration. The absorption peaks of the asymmetric and symmetrical stretching vibrations of the carboxyl group (-COOH) appeared at $1604 \mathrm{~cm}^{-1}$ and $1426 \mathrm{~cm}^{-1}$, respectively. The cluster band at $1150-1000 \mathrm{~cm}^{-1}$ may be said to belong to the various combinations of C-O-C stretching vibrations in the sugar rings (also shown in Figure $3 \mathrm{c}, \mathrm{d}$ ). As regards the spectrum of SA (Figure 3b), it can be discerned that the $-\mathrm{OH}$ stretching vibration is at $3422 \mathrm{~cm}^{-1}$, the $-\mathrm{CH}$ stretching vibration is at $2969-2851 \mathrm{~cm}^{-1}$, and the asymmetric and symmetrical stretching vibration peaks of the carboxyl group are at $1613 \mathrm{~cm}^{-1}$ and $1417 \mathrm{~cm}^{-1}$, respectively. In the spectrum of TA (Figure 3c) a strong absorption band at $1628 \mathrm{~cm}^{-1}$ appeared, which is the stretching vibration of $\mathrm{C}=\mathrm{O}$. The peaks at $1523 \mathrm{~cm}^{-1}$ can be assigned to the bending vibrations of the amino groups. The absorption peaks at 1366 and $1008 \mathrm{~cm}^{-1}$ are due to the asymmetrical and symmetrical stretching, respectively, of the $\mathrm{C}-\mathrm{C}-\mathrm{O}$ system. There are sharp absorption peaks at $900 \sim 650 \mathrm{~cm}^{-1}$ which are mainly caused by trans isomerism vibration. In the spectrum of composite film 4\# (Figure 3d), except for the characteristic absorption peaks of SA and CMCS, the characteristic absorption peaks of the carboxyl group at $1613 \mathrm{~cm}^{-1}$ and $1417 \mathrm{~cm}^{-1}$ have weakened, which verifies that $\mathrm{Ca}^{2+}$ has replaced $\mathrm{Na}^{+}$and crosslinked with the carboxyl group.

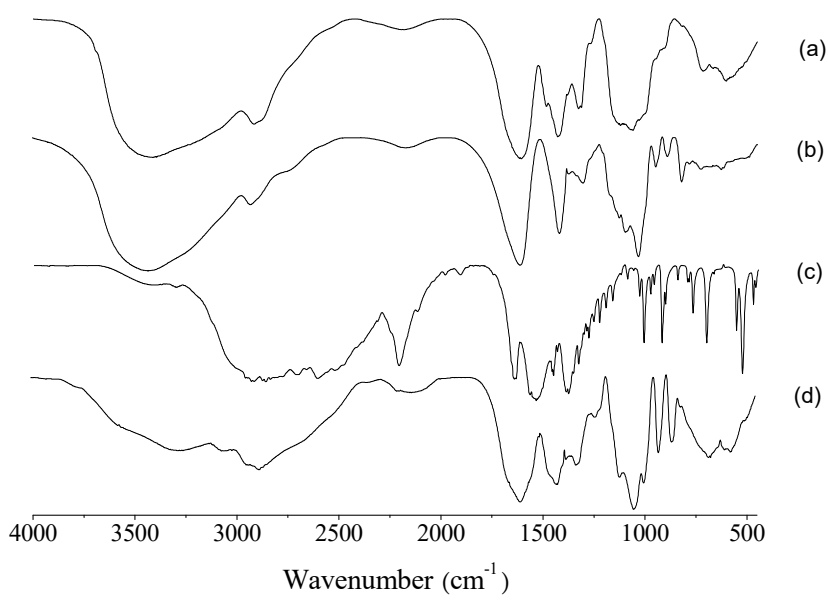

Figure 3. Fourier transform infrared (FTIR) spectra of (a) carboxymethyl chitosan (CMCS), (b) sodium alginate (SA), (c) tranexamic acid (TA), and (d) film $4 \#$. 


\subsection{Cumulative Release Properties}

The cumulative release properties of the composite films were investigated and the results are presented in Figure 4. It can be seen that the cumulative release of tranexamic acid in all the films underwent a similar trend and tended to involve a sudden release within one hour because the drug adsorbed on the surface of the films was first resolved and released. Subsequently, it involved a gentle release tendency after 200 min which was probably due to the drug inside the films being slowly released by diffusion. Film $3 \#$ had the highest cumulative release while film $6 \#$ had the lowest. Crosslinking agents played a key role in the release process. There are many crosslinking agents commonly used in polymer crosslinking such as glyoxal, glutaraldehyde, epichlorohydrin, ionic crosslinking agent, and so on. In the process of preparing the films, a crosslinking agent can be added to the film-forming solution or used in the post-treatment process of the films, which can make the structure of the films more compact and improve the mechanical and barrier properties of the films [20]. However, these chemical crosslinking agents are toxic to some extent, which may cause safety problems and other side effects. In recent years, ionic crosslinking has been used in place of chemical crosslinking [21]. Through ion crosslinking, the performance of films can be significantly improved. This may be because the structure formed by carboxymethyl chitosan and sodium alginate in proper proportions through $\mathrm{Ca}^{2+}$ ionic crosslinking is conducive to the release of tranexamic acid. The cumulative release rate of all the composite films was $\sim 60-80 \%$.

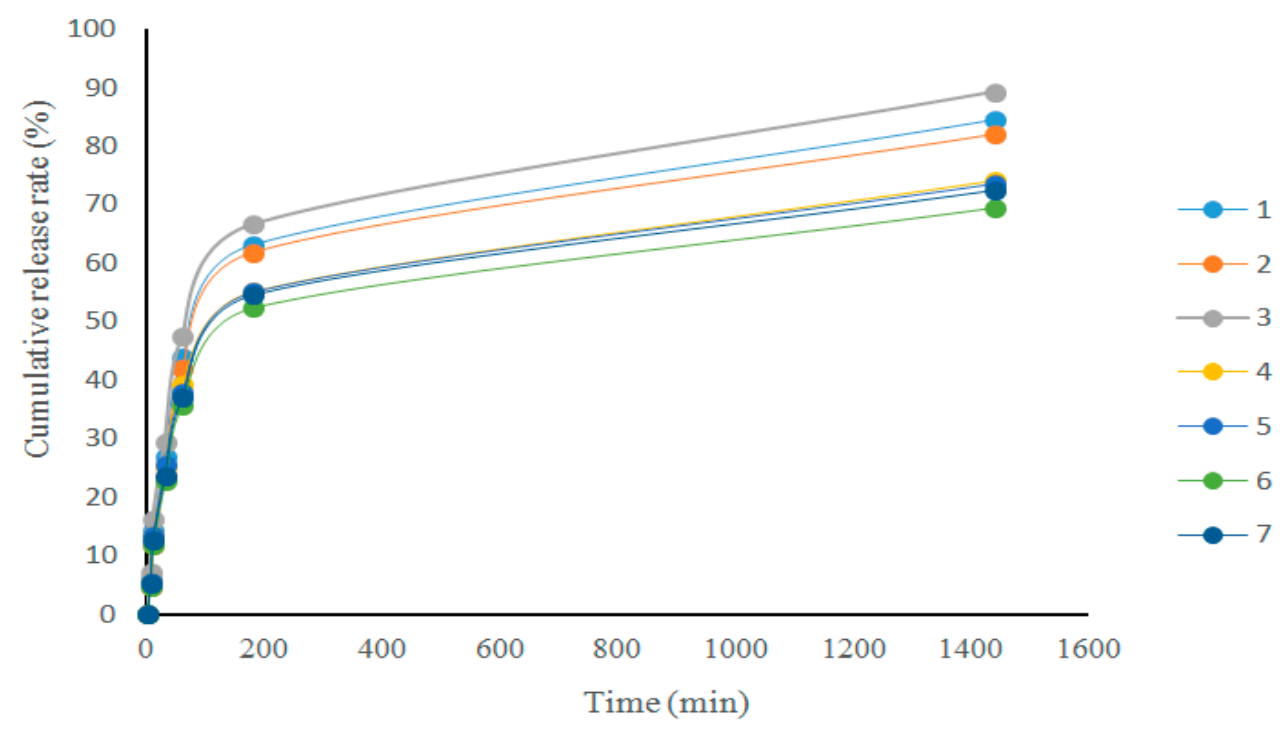

Figure 4. The cumulative release rate of the composite films.

\subsection{Procoagulant Properties}

The procoagulant properties of the composite films in vitro were evaluated by the tube method. The results are shown in Figure 5 and it can be seen that the blood in the blank control group was not coagulated. It appears that film 6\# (pure CMCS) gave similar results to the positive control and film 7\# (pure SA) produced faster clotting, while the mixed films produced even faster clotting times $(<200 \mathrm{~s})$ irrespective of the ratio of SA and CMCS. In particular, film 3\# promoted complete clotting of the rabbit blood within $115 \mathrm{~s}$ and exhibited the best coagulation effect. Films 1\#, 2\#, 4\#, and 5\# also showed excellent procoagulant effects which were superior to that of the positive control. By comparing the results of cumulative release and hemostasis, it can be found that the cumulative release rate of $3 \#$ was high and the time required for hemostasis was short. This may be due to the successful release of tranexamic acid in the composite films. The released tranexamic acid inhibits the lysis of fibrin and accelerated blood coagulation. It may also contact the blood to produce local polymerization, which promotes blood coagulation at the wound, thereby effectively stopping bleeding [22]. 


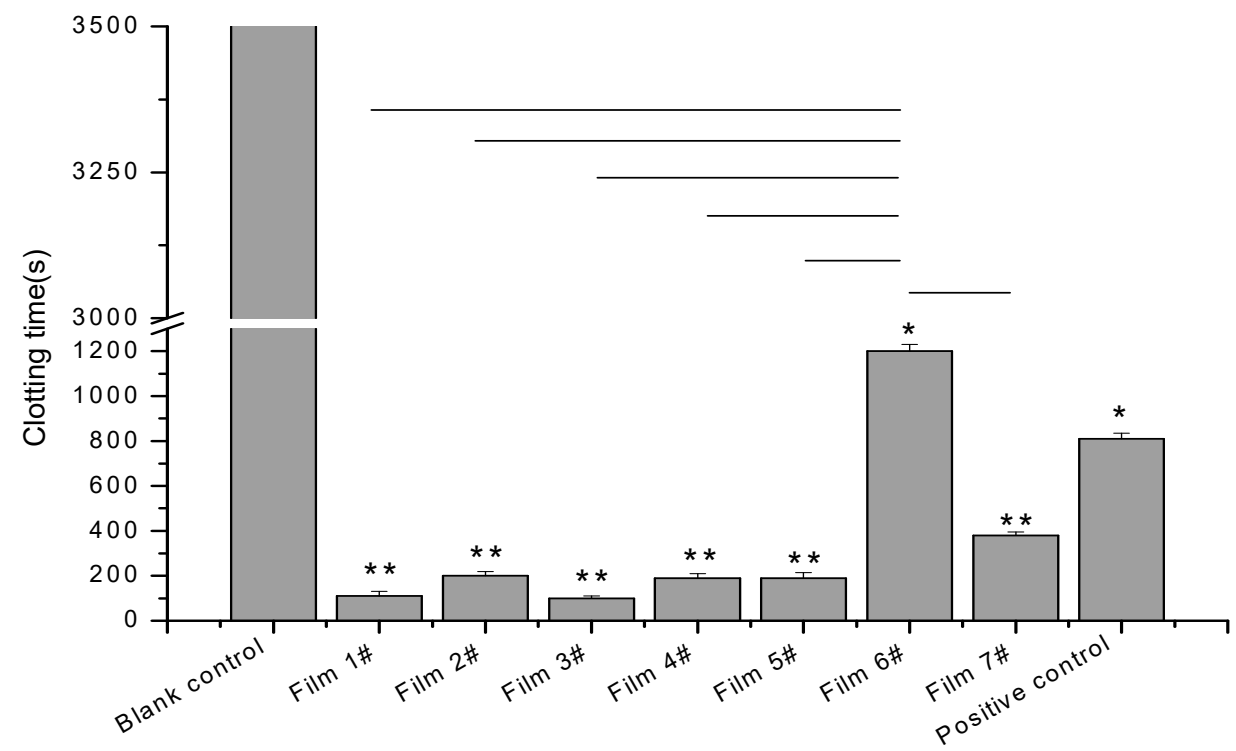

Figure 5. Clotting times of the composite films.

\section{Conclusions}

In this study, carboxymethyl chitosan/sodium alginate/tranexamic acid composite films were successfully prepared by a freeze-drying method. The composite films were porous and had good physical properties which were good for wound healing. The excellent cumulative release properties of the composite films may enhance their hemostatic effects. In particular, film $3 \#$ had the best properties and shows considerable promise for use in the development of hemostatic materials.

Author Contributions: Z.H. and P.-W.L. conceived and designed the study and wrote the manuscript; Q.-K.Z. and Z.-Y.W. performed the experimental work and wrote part of the draft manuscript; and Y.-Q.Q., S.-D.L., and Z.-M.Y. contributed to data analysis and reviewed the manuscript.

Funding: This work was funded by the Guangdong Provincial Natural Science Foundation of China (2016A030308009), the Project of Science and Technology Plan of Guangdong Province (2015A020216019), the Project of Enhancing School with Innovation of Guangdong Ocean University (2017KTSCX090) and the National Guangdong Ocean University Undergraduate Training Programs for Innovation and Entrepreneurship (CXXL2018035).

Acknowledgments: The authors gratefully thank Yifeng Deng (Guangdong Medical University) for his excellent technical assistance.

Conflicts of Interest: The authors declare no conflict of interest.

\section{References}

1. Xu, C.; Xu, F.; Wang, B.; Lu, T.J. Electrospinning of poly(ethylene-co-vinylalcohol) nanofibres encapsulated with Ag nanoparticles for skin wound healing. J. Nanomater. 2011, 2011, 201834. [CrossRef]

2. Al-Qattan, M.M.; Abd-Elwahed, M.M.; Hawary, K.; Arafah, M.M.; Shier, M.K. Myofibroblast expression in skin wounds is enhanced by collagen III suppression. BioMed Res. Int. 2015, 2015, 958695. [CrossRef] [PubMed]

3. Jin, G.; Li, J.; Li, K. Photosensitive semiconducting polymer-incorporated nanofibers for promoting the regeneration of skin wound. Mater. Sci. Eng. C 2017, 70, 1176-1181. [CrossRef] [PubMed]

4. Pogorielov, M.V.; Sikora, V.Z. Chitosan as a hemostatic agent: Current state. Eur. J. Med. Ser. B 2015, 2, 24-33. [CrossRef]

5. Sun, X.; Tang, Z.H.; Pan, M.; Wang, Z.C.; Yang, H.Q.; Liu, H.Q. Chitosan/kaolin composite porous microspheres with high hemostatic efficacy. Carbohydr. Polym. 2017, 177, 135-143. [CrossRef] [PubMed]

6. Yan, X.T.; Tong, Z.R.; Chen, Y.; Mo, Y.H.; Feng, H.Y.; Li, P.; Qu, X.S.; Jin, S.H. Bioresponsive materials for drug delivery based on carboxymethyl chitosan/poly $(\gamma$-glutamic acid $)$ composite microparticles. Mar. Drugs 2017, 15, 127. [CrossRef] [PubMed] 
7. Liu, L.; Lv, Q.; Zhang, Q.Y.; Zhu, H.; Liu, W.; Deng, G.R.; Wu, Y.Q.; Shi, C.F.; Li, H.; Li, L.Z. Preparation of carboxymethyl chitosan microspheres and their application in hemostasis. Disaster Med. Public Health Prep. 2017, 11, 660-667. [CrossRef]

8. Xie, W.; Li, Z.; Yang, M.; Yang, X. Preparation of carboxymethyl chitosan microspheres and the adsorption of heavy metal cadmium. Int. Conf. Innov. Mater. Sci. Tech. 2016, 139, 305-313.

9. Pang, H.T.; Chen, X.G.; Ji, Q.X.; Zhong, D.Y. Preparation and function of composite asymmetric chitosan/CM-chitosan membrane. J. Mater. Sci. Mater. Med. 2008, 19, 1413-1417. [CrossRef] [PubMed]

10. Horibe, S.; Tanahashi, T.; Kawauchi, S.; Mizuno, S.; Rikitake, Y. Preventative effects of sodium alginate on indomethacin-induced small-intestinal injury in mice. Int. J. Med. Sci. 2016, 13, 653-663. [CrossRef]

11. Shi, X.Y.; Fang, Q.; Ding, M.; Wu, J.; Ye, F.; Lv, Z.B.; Jin, J. Microspheres of carboxymethyl chitosan, sodium alginate and collagen for a novel hemostatic in vitro study. J. Biomater. Appl. 2016, 30, 1092-1102. [CrossRef]

12. Zhong, H.Q.; Ye, W.J.; Li, X.Y.; Wang, X.Y.; Sun, R.C. Polyelectrolyte sponge reinforced with organic rectorite and silver nanoparticles. Curr. Nanosci. 2013, 9, 742-746. [CrossRef]

13. Montroy, J.; Hutton, B.; Moodley, P.; Fergusson, N.A.; Cheng, W.; Tinmouth, A.; Lavallée, L.T.; Fergusson, D.A.; Breau, R.H. The efficacy and safety of topical tranexamic acid: A systematic review and meta-analysis. Trans. Med. Rev. 2018, 32, 165-178. [CrossRef]

14. Sard, S.; Errassifi, F.; Marsan, O.; Geffre, A.; Trumel, C.; Drouet, C. Adsorption of tranexamic acid on hydroxyapatite: Toward the development of biomaterials with local hemostatic activity. Mater. Sci. Eng. C 2016, 66, 1-7. [CrossRef] [PubMed]

15. Zareh, M.M.; Wassel, A.A.; Abd Alkarem, Y.M. Electrochemical sensors for determination of tranexamic acid in pure form and pharmaceutical preparations. Int. J. Pharm. Sci. Res. 2016, 1, 5-12.

16. Cheng, F.; Liu, C.Y.; Li, H.B.; Wei, X.J.; Yan, T.S.; Wang, Y.F.; Song, Y.J.; He, J.M.; Huang, Y.D. Carbon nanotube-modified oxidized regenerated cellulose gauzes for hemostatic applications. Carbohydr. Polym. 2018, 183, 246-253. [CrossRef]

17. Balakrishnan, B.; Soman, D.; Payanam, U.; Laurent, A.; Labarre, D.; Jayakrishnan, A. A novel injectable tissue adhesive based on oxidized dextran and chitosan. Acta Biomater. 2017, 53, 343-354. [CrossRef]

18. Luo, P.F.; Nie, M.; Wen, H.G.; Xu, W.Y.; Fan, L.H.; Cao, Q.H. Preparation and characterization of carboxymethyl chitosan sulfate/oxidized konjac glucomannan hydrogels. Int. J. Biol. Macromol. 2018, 113, 1024-1031. [CrossRef] [PubMed]

19. Wang, C.; Luo, W.F.; Li, P.W.; Li, S.D.; Yang, Z.M.; Hui, Z.; Liu, Y.Y.; Ao, N.J. Preparation and evaluation of chitosan/alginate porous microspheres/Bletilla striata polysaccharide composite hemostatic sponges. Carbohydr. Polym. 2017, 174, 432-442. [CrossRef] [PubMed]

20. Murphy, R.W.; Farkas, B.E.; Jones, O.G. Effect of crosslinking on the physical and chemical properties of $\beta$-lactoglobulin (Blg) microgels. J. Colloid Interface Sci. 2017, 505, 736-744. [CrossRef] [PubMed]

21. Hui, L.; Gao, C. Preparation and properties of ionically cross-linked chitosan nanoparticles. Polym. Adv. Technol. 2010, 20, 613-619.

22. Wang, J.; Hao, S.L.; Luo, T.T.; Yang, Q.; Wang, B.C. Development of feather keratin nanoparticles and investigation of their hemostatic efficacy. Mater. Sci. Eng. C 2016, 68, 768-773. [CrossRef] [PubMed]

(C) 2019 by the authors. Licensee MDPI, Basel, Switzerland. This article is an open access article distributed under the terms and conditions of the Creative Commons Attribution (CC BY) license (http:// creativecommons.org/licenses/by/4.0/). 